\title{
Guest-host supramolecular assembly of injectable hydrogel nanofibers for cell encapsulation
}

Beverly Miller ${ }^{1}$, Audrey Hansrisuk ${ }^{2}$, Christopher B. Highley ${ }^{1,3}$, Steven R. Caliari ${ }^{1,3, *}$

${ }^{1}$ Department of Chemical Engineering, University of Virginia, Charlottesville, Virginia 22904

${ }^{2}$ Department of Chemistry, University of Virginia, Charlottesville, Virginia 22904

${ }^{3}$ Department of Biomedical Engineering, University of Virginia, Charlottesville, Virginia 22904

* Corresponding author: caliari@ virginia.edu

Supplemental Figures:
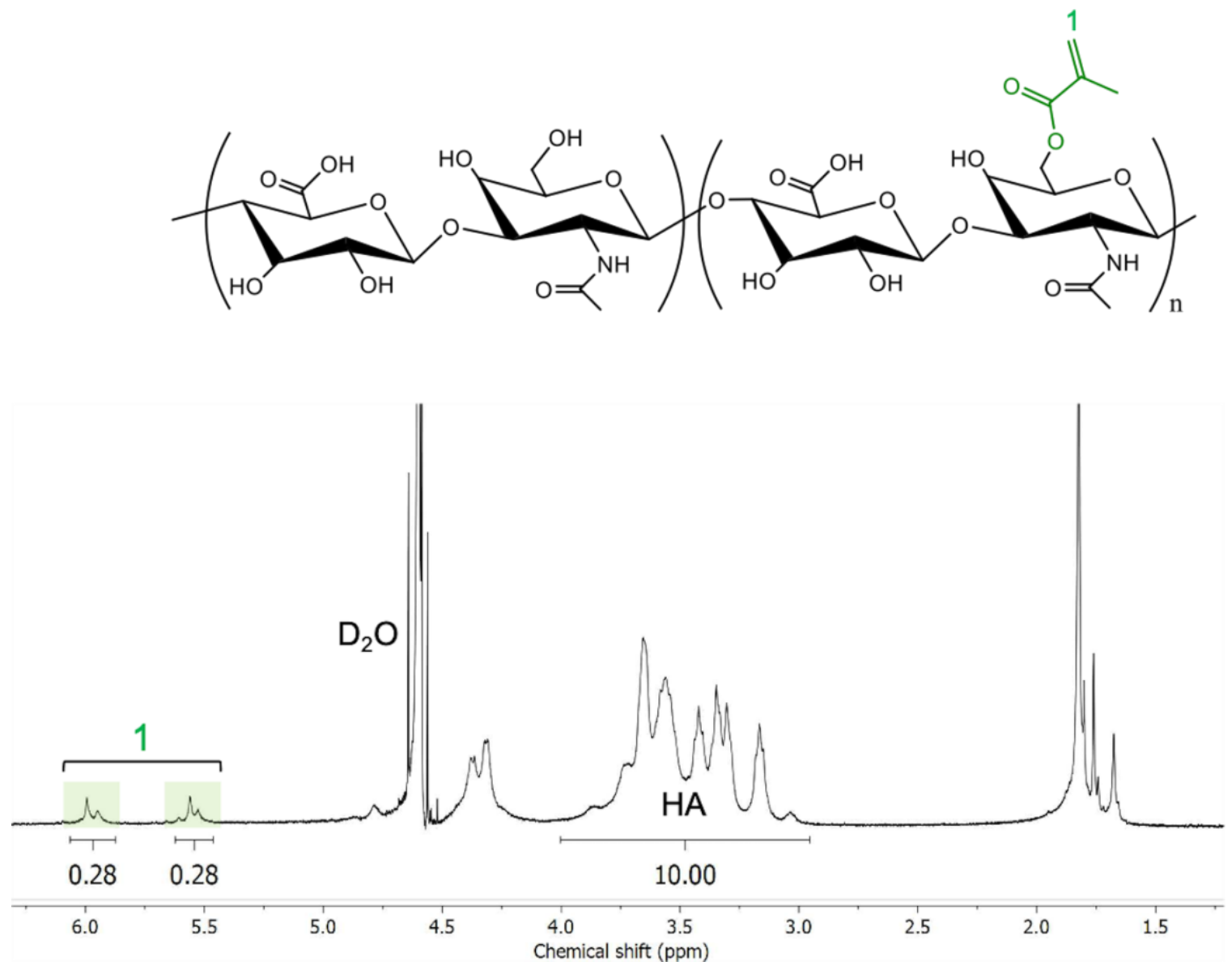

Figure S1. ${ }^{1} \mathrm{H}$ NMR spectrum of methacrylate-modified hyaluronic acid (MeHA). The degree of HA modification with methacrylates was determined to be $28 \%$. Degree of functionalization was determined from integration of the methylene $(\delta=5.82,1 \mathrm{H}$ and $\delta=6.25,1 \mathrm{H})$ highlighted green and labeled ' 1 ' relative to the HA backbone, $(\delta=3.10-4.10,10 \mathrm{H})$. 


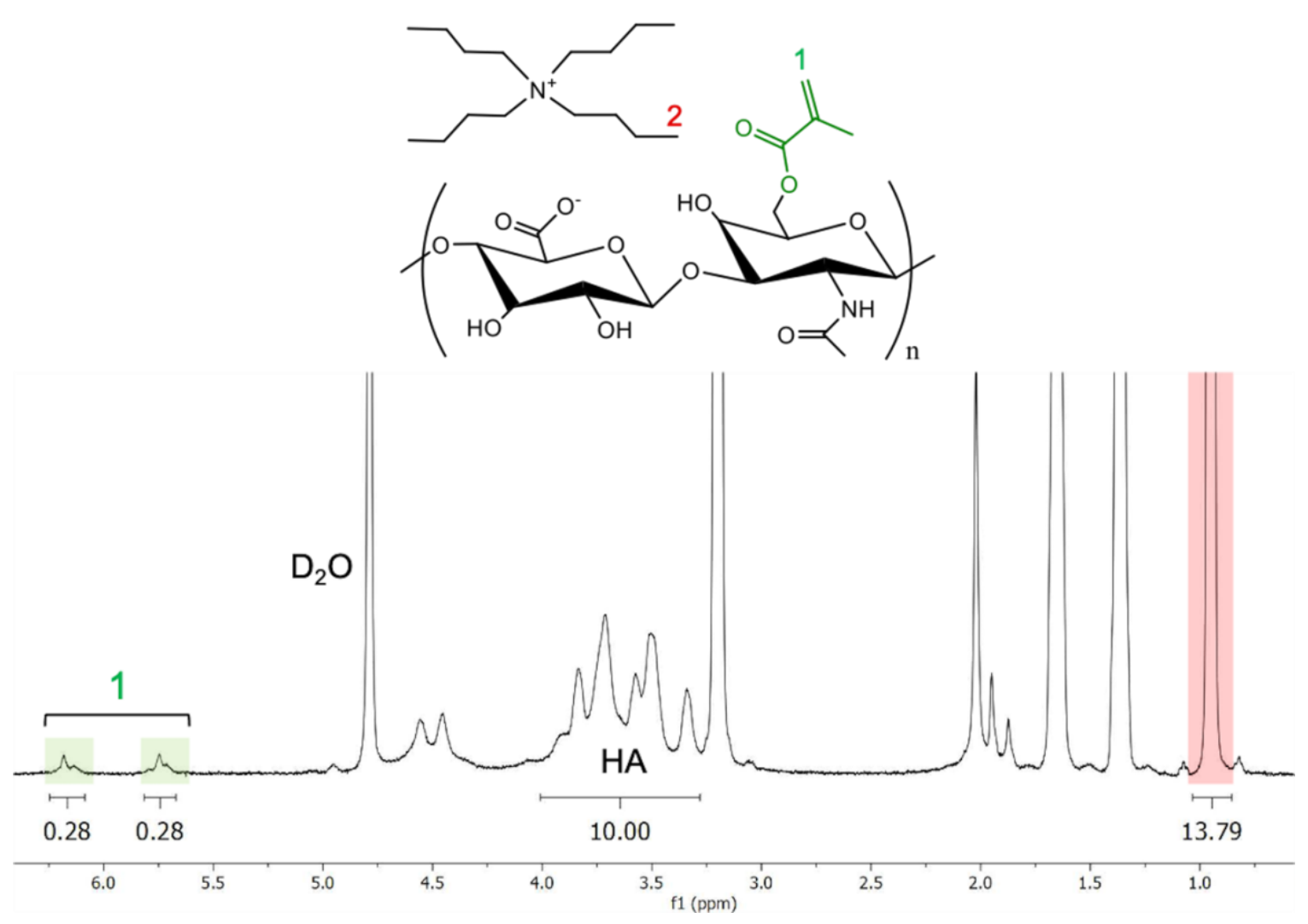

Figure S2. ${ }^{1} \mathrm{H}$ NMR spectrum of methacrylated hyaluronic acid tert-butyl ammonium salt (MeHA-TBA). The MeHA used for the synthesis of Ad (guest) and CD (host) derivatives underwent addition of the TBA salt (highlighted red) to enable further HA modification. 


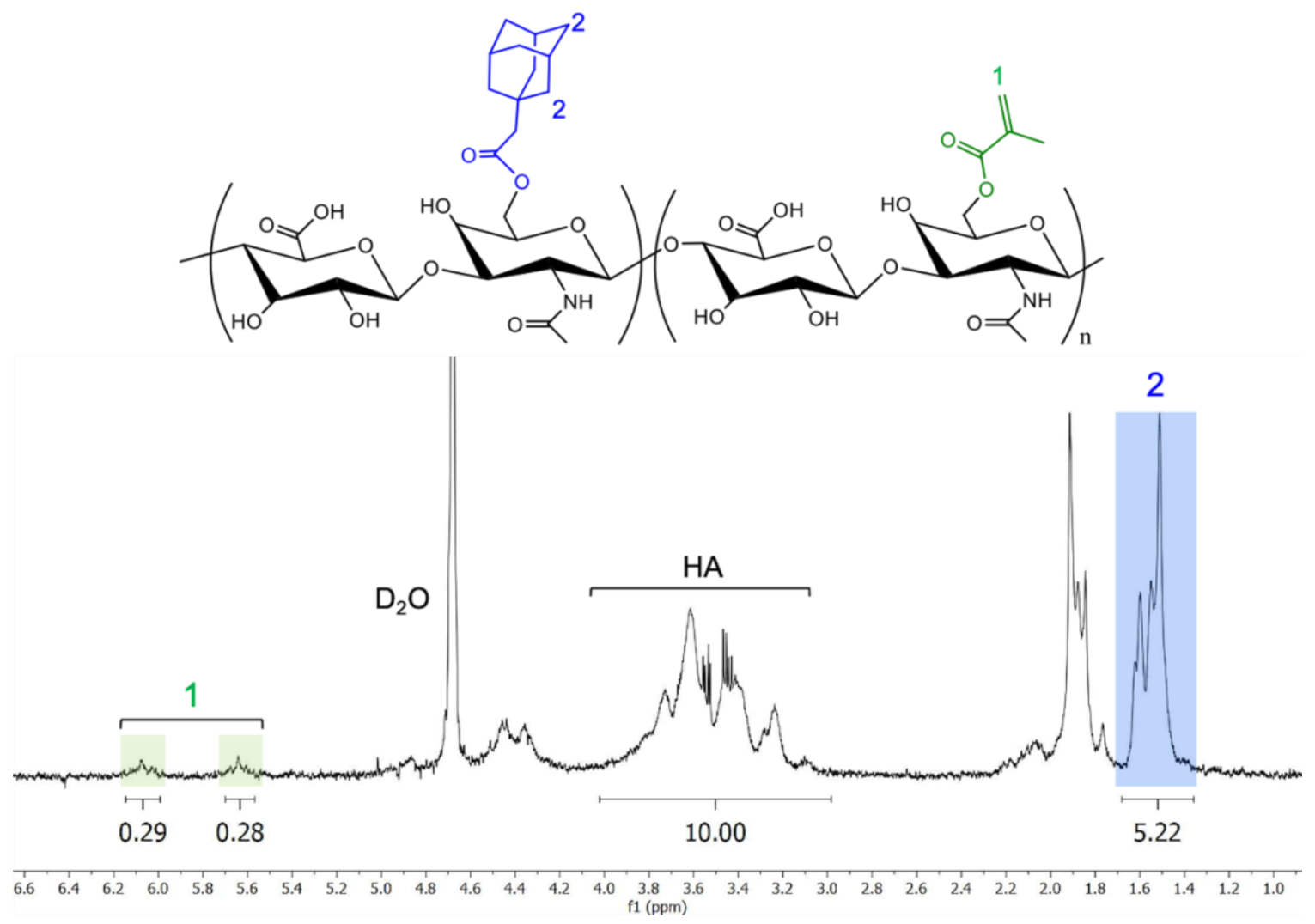

Figure S3. ${ }^{1} \mathrm{H}$ NMR spectrum of adamantane and methacrylate-modified hyaluronic acid (Ad-MeHA). The degree of MeHA modification with adamantane was determined to be $43 \%$. The integration of the ethyl multiplet of adamantane $(\delta=1.40-1.70,12 \mathrm{H})$ highlighted in blue gives the degree of modification relative to the HA backbone $(\delta=3.10-4.10,10 \mathrm{H})$. 

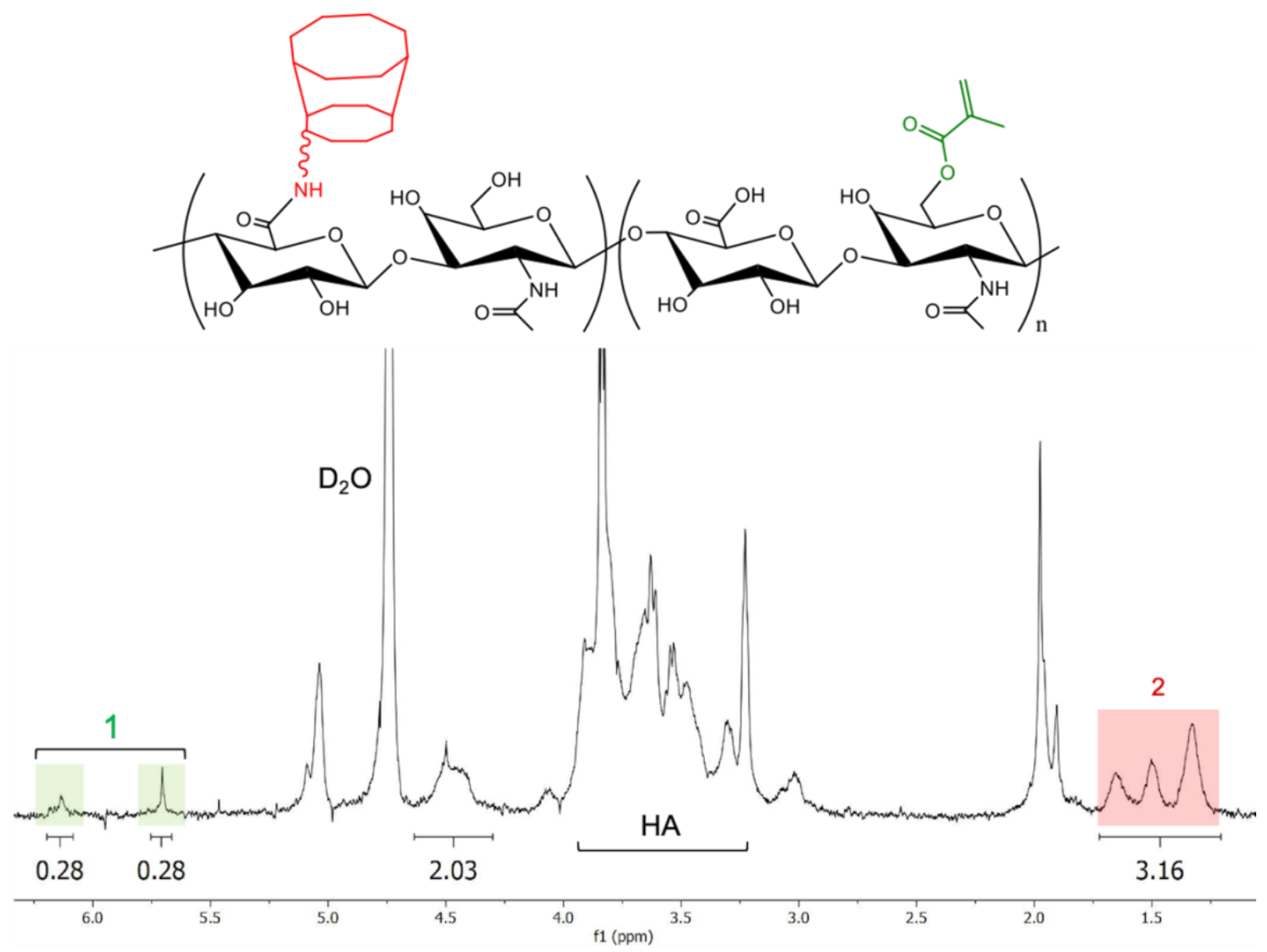

Figure S4. ${ }^{1} \mathrm{H}$ NMR spectrum of $\beta$-cyclodextrin and methacrylate-modified hyaluronic acid (CD-MeHA). The degree of MeHA modification with $\beta$-cyclodextrin was determined to be $26 \%$. This was calculated from the integration of the hexane linker $(\delta=1.20-1.75,12 \mathrm{H})$ highlighted in red relative to the methacrylate modification, which is assumed to stay constant. 

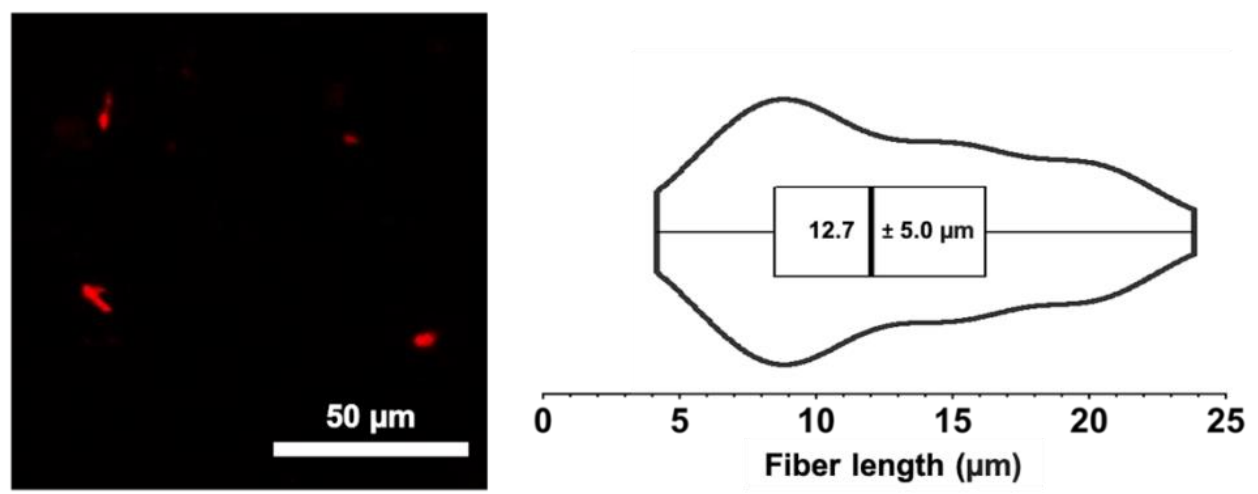

Figure S5. Fiber length distribution following trituration. The lengths of rhodamine-labeled Ad-MeHA 'guest' and CD-MeHA 'host' fibers were separately evaluated following sequential needle trituration. Fiber lengths $(n=90)$ were measured using confocal images and processed in ImageJ. A representative image of Ad-MeHA guest fibers is shown here. 


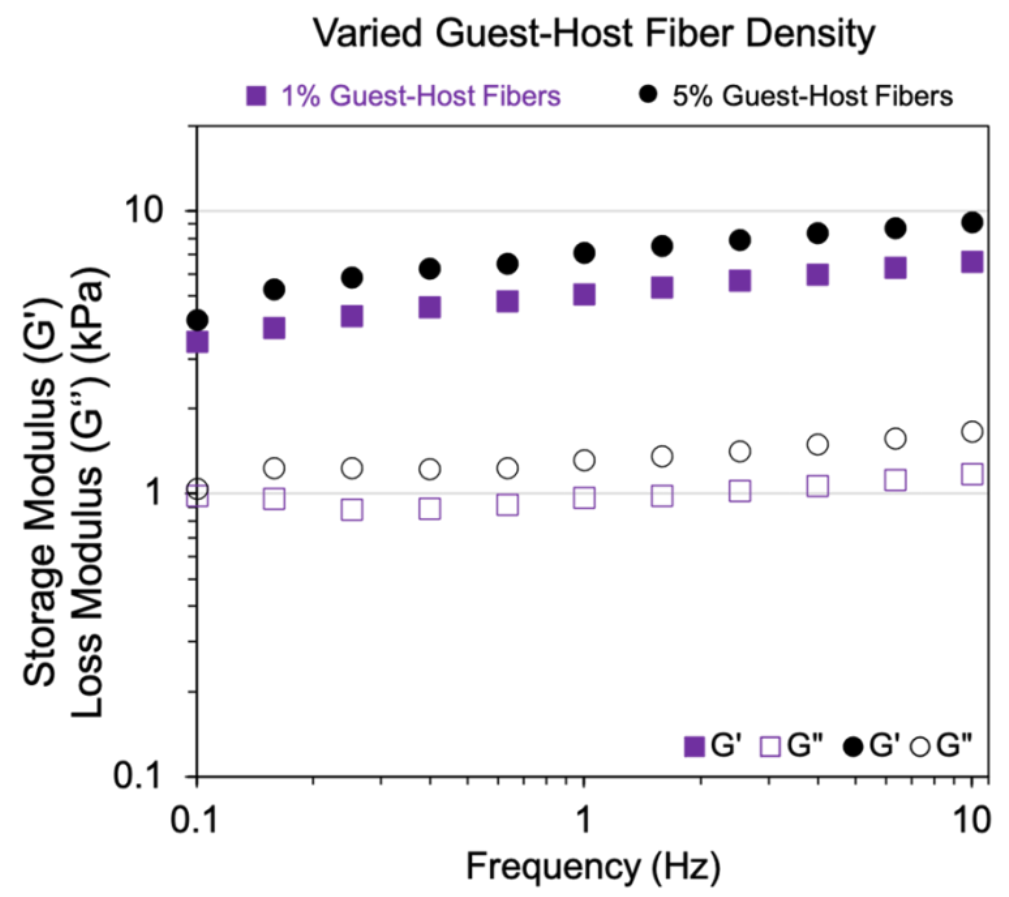

Figure S6. Rheological properties of the guest-host fiber network with varying fiber density. The frequency-dependent behavior was measured using a constant strain of $0.5 \%$. The $1 \%$ fibrous hydrogel reached a final storage modulus $\left(\mathrm{G}^{\prime}\right)$ of $6.6 \mathrm{kPa}$ and the $5 \%$ fibrous hydrogel a $\mathrm{G}^{\prime}$ of 9.2 $\mathrm{kPa}$ at $10 \mathrm{~Hz}$. 


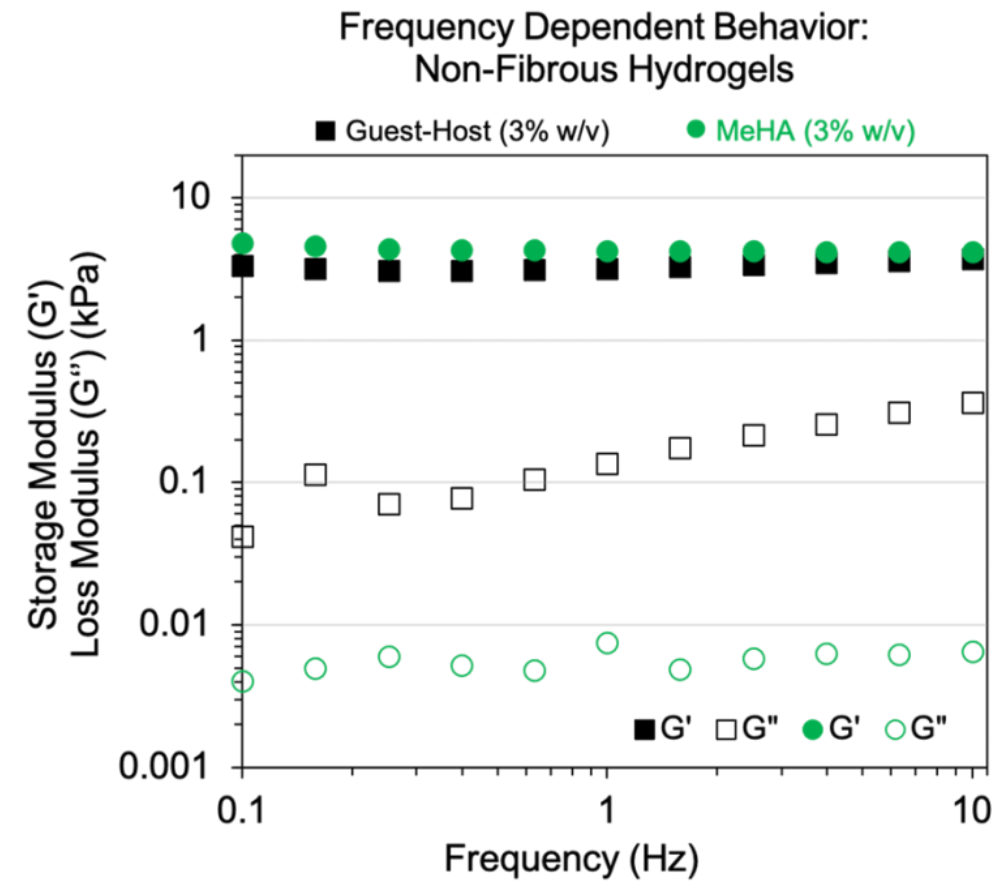

Figure S7. Rheological properties of the non-fibrous MeHA and guest-host hydrogels used for cell encapsulation. The frequency-dependent behavior was measured using a constant strain of $0.5 \%$. The non-fibrous $3 \% \mathrm{MeHA}$ hydrogel formulation reached a final storage modulus $\left(\mathrm{G}^{\prime}\right)$ of $4.2 \mathrm{kPa}$ and the non-fibrous $3 \%$ guest-host hydrogel formulation reached a final $\mathrm{G}^{\prime}$ of $3.7 \mathrm{kPa}$ at $10 \mathrm{~Hz}$. 

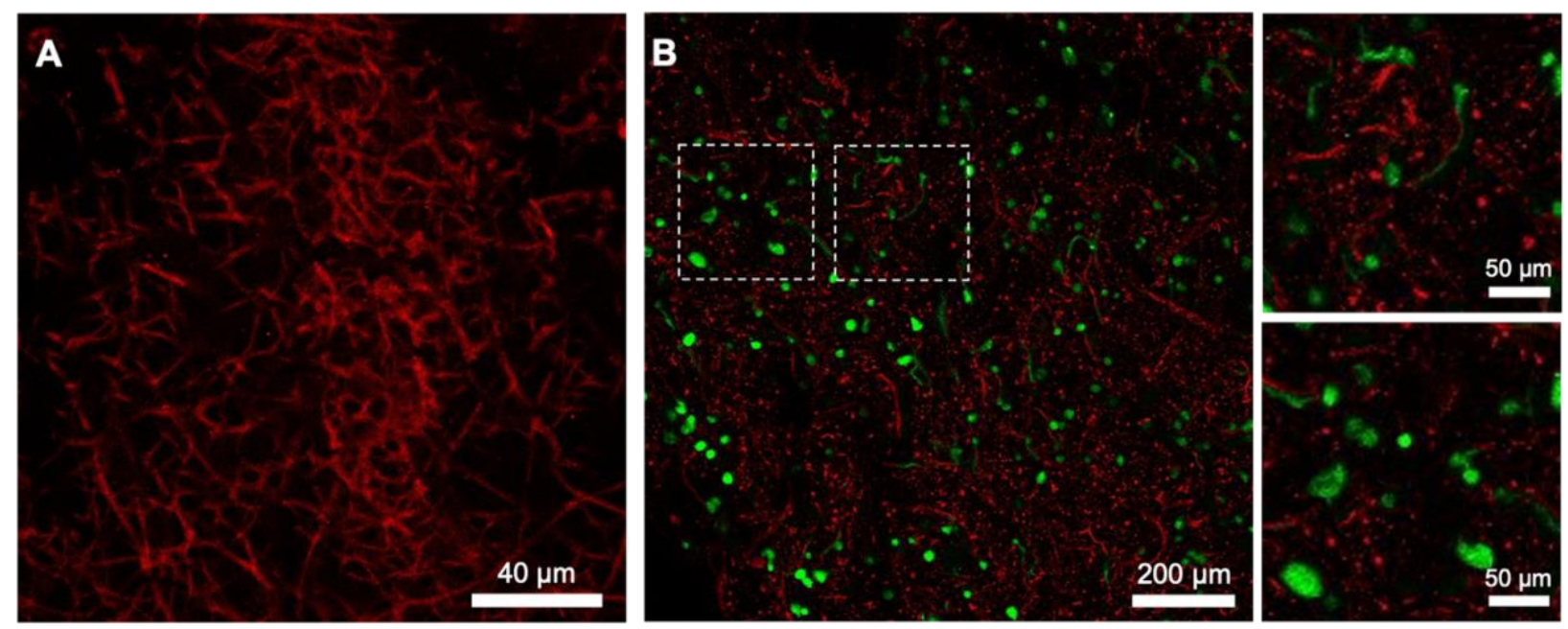

Figure S8. Visualization of the fibrous guest-host hydrogel structure. Rhodamine-labeled HA fibers show A) the morphology of the fibrous guest-host hydrogel ( $r e d$ ) without encapsulated cells and B) hMSCs (green) encapsulated within the fibrous guest-host hydrogel (red) after 7 days of culture. 